Número especial "Convenção internacional sobre meio ambiente e desenvolvimento para integração e cooperação para a sustentabilidade” - Havana/Cuba.

Vol. 25, n. 1, 2020.

Artigo recebido em: 03/02/2020

Artigo aprovado em: 04/03/2020

\title{
LA TEMPORALIDAD DE LA PARTICIPACIÓN Y VALOR DE SU CONSIDERACIÓN PARA LA SOSTENIBILIDAD AMBIENTAL DEL HÁBITAT EN PEQUEÑAS COMUNIDADES COSTERAS
}

A TEMPORALIDADE DA PARTICIPAÇÃO E A IMPORTÂNCIA DE SUA CONSIDERAÇÃO PARA A SUSTENTATIBILIDADE AMBIENTAL DO HABITAT EM PEQUENAS COMUNIDADES COSTEIRAS

\section{THE TEMPORALITY OF PARTICIPATION AND VALUE OF ITS CONSIDERATION FOR THE ENVIRONMENTAL SUSTAINABILITY OF THE HABITAT IN SMALL COASTAL COMMUNITIES}

\author{
Joaquin Alonso Freyre ${ }^{1}$ \\ Ginley Durán Castellón ${ }^{2}$ \\ Edelkis Rodríguez Moya ${ }^{3}$
}

\begin{abstract}
RESUMEN
La consideración de la temporalidad de la participación para la sostenibilidad ambiental del hábitat en pequeñas comunidades costeras resulta indispensable por su aporte a la diferenciación del encargo que corresponde a cada sujeto implicado en tal sostenibilidad, al diagnóstico del estado de simetría social presente en sus relaciones sociales mutuas y al planteo de alternativas de solución de problemáticas con el involucramiento de todos los implicados. Ello es un requerimiento de gobernanza de la sostenibilidad ambiental del hábitat para afrontar el cambio climático como ocurre con la reubicación espacial de comunidades con decisiones que impactan de manera profunda en su vida cotidiana, no solo en el ejercicio laboral relacionado con la costa, sino además en toda la subjetividad involucrada con el arraigo al ambiente marino, donde los afectados son atendidos solo en términos de percepción del riesgo.
\end{abstract}

Palabras clave: participación, temporalidad, sostenibilidad ambiental, hábitat y comunidades costeras.

\footnotetext{
${ }^{1}$ Universidad Central "Marta Abreu" de Las Villas, Cuba, Correo: joaquin@uclv.edu.cu;

2 Universidad Central "Marta Abreu" de Las Villas, Cuba, Correo: ginleyd@uclv.edu.cu;

${ }^{3}$ Centro de Estudios y Servicios Ambientales Villa Vlara (CESAMVC), Cuba, Correo: director@cesam.vcl.cu.
} 


\section{ABSTRACT}

The need to assume participation considering the project that gives directionability and the differentiation of involved subjects has been stablished by the Center of Community Studies from UCLV as a superior stage of its approach. Its logical consequence is to incorporate temporality as an analytical dimension within the differentiation because temporary succession does not concern all the subjects from the social logic that is considered at the time it allows to stablish a participation typology of condition that shows the state of the social symmetry present in the process, correlating in a data matrix empiric and temporal distribution. Indicators of temporary succession as demand, decision, realization and control are stablished for the Project. The situation of the small coastal communities makes up a scope of implementation where application is governance requirement of habitat environmental sustainability given that the projections of spatial relocation planned for them, and in some cases done, is made at many kilometers from the initial location at shore line, deeply impacts their daily lives, not only in the jobs exercise related to the coast but also in the whole subjectivity linked to the marine environment root. Even more when the decisions are taken at many kilometers far and of social structure, in a process of exclusion in which the affected people are considered only in terms of danger, risk and vulnerability.

Keywords: participation, temporality, sustainability, communities.

\section{INTRODUCCIÓN}

"Participación" es un término de muy amplio uso en la literatura política, científica y comercial en la actualidad. El Centro de Estudios Comunitarios (CEC) de la Universidad Central de Las Villas en Cuba lo ha venido empleando dentro de su concepción de comunidad cuando la define como grupo social donde transcurren procesos de participación y cooperación en torno a un proyecto colectivo (Colectivo de autores, 2016: 21) en una relación categorial que vincula a la participación con la proyección que le da significación y sentido.

Tal vínculo resulta esencial en la superación de un tratamiento abstracto de la participación pues se asume incorporando lo que direcciona su realización ya que las personas y grupos humanos no participan en general, sino para el logro de algo que compulsa a la actuación colectiva, donde cada sujeto implicado en la actividad social aporta desde la función específica que le corresponde desempeñar dentro del proyecto, bajo la mediación que proviene de la expectativa socialmente configurada para tal función, es decir, la función 
mediada por el rol por lo que en su integridad están presentes lo objetivo y lo subjetivo.

Ello ha permitido al CEC argumentar el postulado de la participación diferenciada (Alonso y Jara, 2016) que parte de dos definiciones: 1participación como involucramiento activo, individual o colectivo, de las personas como sujetos de la actividad y 2- proyecto como sistema de acción que contiene un objeto, una finalidad y unos medios de actuación.(: 4) La integración de los elementos objetivos y subjetivos presentes en estas definiciones conduce a "la idea de la necesidad de diferenciar el aporte que cada sujeto tipo tributa al proyecto desde lo que le corresponde hacer como encargo social (estructura, función y significación) para el despliegue de la actividad conjunta" ya que "no se toman a los sujetos en su singularidad, sino como tipos sociales que se involucran desde la relación social específica a través de la cual se produce tal actividad" (: 5) La distinción que se hace en cuanto a que no a todos los sujetos implicados en la actividad les corresponde el mismo encargo participativo, permite plantear la categoría de participación diferenciada.

\section{LA TEMPORALIDAD EN LA DIFERENCIACIÓN DE LA PARTICIPACIÓN}

Considerar la relación categorial entre participación y proyecto trae otra consecuencia de naturaleza heurística: la participación no transcurre en un acto, sino a través de un proceso que se evidencia en etapas. Al respecto es necesario considerar el devenir de los procesos decisorios dentro de los proyectos que estructuran la actividad humana.

En un estudio realizado Del Río (2002) sobre el proceso de toma de decisiones públicas a partir de una significativa revisión de autores, se llega a las siguientes las fases de tal proceso:

1) identificación del problema,

2) búsqueda de alternativas de solución,

3) selección de la alternativa óptima, o toma de la decisión,

4) ejecución o implementación de la decisión, y 
5) control de la ejecución de la decisión.

Otro autor, Branko Horvart (citado por Dilla, 1993: 83), distingue cuatro fases en cualquier proceso decisorio: Fase agregativa (momento de definición de los problemas), Fase convertiva (proceso de búsqueda de alternativas y selección de la más apropiada), Fase implementativa (momento de implementación o ejecución de la decisión), y Fase evaluativa (evaluación del resultado obtenido a partir de la ejecución de la decisión tomada). Tales fases han sido consideradas por Caballero y Yordy (2004) como "etapas de participación según la secuencia de acciones en que participa la población". (: 161)

Desde estos antecedentes es posible plantear en la perspectiva de temporalidad las siguientes etapas de participación considerando la proyección que la direcciona: demanda, decisión, ejecución, impacto, control. A su vez, al interior de cada etapa hay una temporalidad a considerar para explicar y comprender la participación. 


\begin{tabular}{|c|c|}
\hline \multicolumn{2}{|c|}{ Temporalidad de la participación por etapas y procesos } \\
\hline Etapas & Procesos \\
\hline 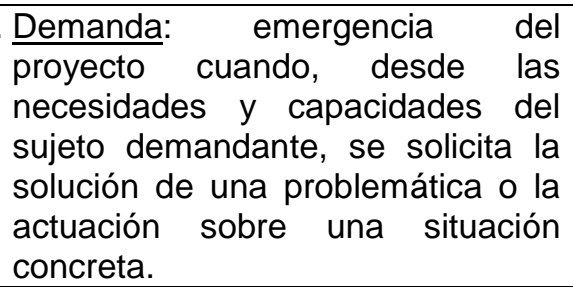 & $\begin{array}{l}\text { El movimiento de la necesidad a la demanda pasa por } \\
\text { intereses y capacidades del sujeto demandante para } \\
\text { interpretar la necesidad y formular la solicitud, incluyendo } \\
\text { los términos que emplea. }\end{array}$ \\
\hline $\begin{array}{l}\text { Decisión: determinación } \text { de } \\
\text { acciones a ejecutar en la práctica } \\
\text { en respuesta a lo demandado. }\end{array}$ & $\begin{array}{l}\text { La decisión sobre lo demandado se realiza mediante la } \\
\text { formulación de alternativas, su confrontación y la toma de } \\
\text { decisión en correspondencia con las relaciones de poder } \\
\text { imperantes dentro del campo de actividad en que } \\
\text { transcurre la participación. }\end{array}$ \\
\hline $\begin{array}{llr}\text { Ejecución: } & \text { gestión de } \\
\text { implementación de lo decidido } \\
\text { mediante una secuencia de } \\
\text { acciones concretas. }\end{array}$ & $\begin{array}{l}\text { La ejecución transcurre mediante relaciones entre } \\
\text { sujetos mediadas por el estado de los medios y objetos } \\
\text { de la actividad, por el estado de simetría dentro del } \\
\text { campo y por los fines de la participación. }\end{array}$ \\
\hline $\begin{array}{l}\text { Impacto: efecto de lo ocurrido con } \\
\text { la demanda y con los sujetos } \\
\text { implicados en el proyecto } \\
\text { incluyendo la apropiación de la } \\
\text { experiencia y sus resultados. }\end{array}$ & $\begin{array}{l}\text { El impacto objetivo y subjetivo del proceso participativo } \\
\text { comienza desde la propia emergencia del proyecto al } \\
\text { plantearse la solicitud, lo que ocurre o no con lo } \\
\text { solicitado, lo decidido, lo ejecutado y lo demandado, aun } \\
\text { cuando se ubique como cuarta etapa para propiciar la } \\
\text { retrospectiva sobre el asunto. }\end{array}$ \\
\hline $\begin{array}{lll}\text { Control: } & \text { Verificación } & \text { de } \\
\text { cumplimiento de objetivos } & \text { y } & \text { de } \\
\text { ejecución de lo decidido. } & & \end{array}$ & $\begin{array}{l}\text { El control se realiza en cada momento del proyecto } \\
\text { (control de proceso) y al concluir (control de resultados). } \\
\text { Según quien lo ejecuta se adjetiva, por ejemplo, } \\
\text { considerando los sujetos tipos generales existe el control } \\
\text { gubernamental, popular y científico. }\end{array}$ \\
\hline
\end{tabular}

Fuente: Autores

La condición desde la cual cada sujeto implicado participa, considerando la lógica del campo de la actividad social del proyecto (vía deductiva) y los resultados de investigaciones realizadas (vía inductiva) con aplicación de la concepción de diferenciación de la participación (Jara, 2015 y Durán, 2018) puede ser identificada en la siguiente tipología de sujetos: demandante, decisor, ejecutor, destinatario y apoyo.

Una definición simple de cada uno de naturaleza operacional puede darse del siguiente modo:

- Demandante: solicita el proyecto

- Decisor: decide si se satisface o no la demanda y como.

- Ejecutor: implementa lo decidido

- Destinatario: beneficiario del proyecto 
- Controlador: verifica el cumplimiento de lo decidido durante el proceso y en resultados

- Apoyo: interviene en el proyecto mediante una(s) acción(es) puntual(es).

La condición de cualquier sujeto proviene de lo que Bourdieu (1988) ha planteado como "determinantes sociales de las prácticas" (: 27) que un investigador debe considerar para superar la ilusión de libertad que se pueda tener respecto a tales determinaciones. De ahí la necesidad de asumir la condición de cada sujeto como el límite y la posibilidad de actuación que tiene en el campo de la actividad donde participa. Las personas y grupos humanos en tanto sujetos no son demandantes, decisores o cualquier otra condición posible dentro del proyecto por libre elección, sino como resultante de las determinaciones presentes en dicho campo de actividad. Ejemplo, la determinación social de los excluidos los limita de plantear demandas, tomar decisiones, etc. respecto a problemáticas o situaciones que los afecta.

De ahí la necesidad de establecer dos registros de diferenciación de la participación en una tabla (en nuestra experiencia utilizando Excel) donde se ubiquen en las filas los sujetos tipo agrupados en sujetos generales (gobierno, comunidad y academia) y en las columnas la sucesión de acciones agrupadas en etapas. Un primer registro para colocar los datos de una distribución teórica de la participación según la lógica de lo socialmente necesario y posible; un segundo registro con la distribución empírica de los datos de la participación existente.

El dato que se coloca en cada celda hace referencia a la participación real (uno) o no (cero) de cada sujeto en cada acción. Según la distribución de que se trate, se hace una afirmación sobre la condición debida o real por sujetos y por acciones. Su comparación tiene una salida práctica que aporta no solo al diagnóstico del estado de cosas existentes respecto a la participación que se indaga, sino además, propositiva sobre aquello que debe ser cambiado para afrontar problemáticas con el involucramiento de todos los que deban estar implicados. 
Para sostenibilidad ambiental del hábitat en pequeñas comunidades costeras esta es una cuestión relevante pues se trata de un asunto que impacta de modo permanente en la vida de toda la comunidad y de cada uno.

\section{PEQUEÑAS COMUNIDADES COSTERAS EN CUBA}

Con la creación del Panel Intergubernamental para el Cambio Climático en el año 1998 y a partir de la compilación de resultados de investigación, la modelación de escenarios futuros y sus subsecuentes informes, que ya llegan a cinco, surgen en la escena científica los primeros enunciados sobre el cambio climático que experimenta el planeta al transgredir limites esenciales de la estabilidad atmosférica (IPCC, 2013).

Uno de los elementos abordados por estos informes es el hecho indiscutible ya de la subida progresiva del nivel medio del mar, que por lógica afecta y pone en riesgo a los estados insulares con mayor incidencia. Daños significativos experimentan los ecosistemas del borde litoral, en especial las playas que son erosionadas significativamente (Juanes y col., 2014).

Fundamentalmente se afectan las islas del cinturón tropical y subtropical del planeta, donde por los efectos sumatorios de la dilatación de la masa marina, a causa del aumento de la temperatura global, y el derretimiento de los hielos polares y glaciares, el océano incrementa sus cotas de inundación costera en zonas bajas, por penetraciones de marea, incrementada por la surgencia ante eventos meteorológicos extremos.

En contraste con los estados continentales, los Pequeños Estados Insulares en Desarrollo tienen un recurso básico sumamente limitado y vulnerable ante los efectos del cambio climático: la tierra emergida. (UNEP, 2008).

Es precisamente en este presupuesto físico en el que el peligro, la vulnerabilidad y el riesgo de las comunidades costeras de zonas bajas, dispara primeramente las alarmas ambientales y por tanto las alarmas sociales, proceso que debe ocurrir a la par o a la inversa, pero que en el estado actual de cosas tiene esta ruta crítica. 
Desafortunadamente aún, los análisis de los efectos e impactos sociales muchas veces no se ven como el hecho de mayor peso si no secundario, por detrás de los daños infraestructurales, ecosistémicos y de pérdidas económicas.

El término "desastre" se aplica con mayor incidencia a la pérdida de recursos de sectores productivos, de servicios, daños a viviendas e infraestructura construida y no a las profundas huellas y cicatrices psicosociales que quedan en los pobladores que subsisten.

Entran en prolongados períodos de readaptación y recuperación de su estabilidad vital. En el peor de los casos incluye el hecho de recuperar una vivienda donde reestablecer su ámbito vital y volver a adquirir sus artículos y enseres.

Con frecuencia en estos eventos se pierde también la memoria archivística de las familias y por lo tanto de gran parte de las imágenes y sonidos del pasado de las comunidades, perdiéndose a veces la trazabilidad del decursar de los años de grupos sociales.

Surge en este suceso de reacomodo familiar, y se potencia, un nuevo concepto: desplazados climáticos. Concepto este acompañado de impactos sociales significativos, al planificarse y ejecutarse movimientos masivos y reubicación de personas con poca o nula consulta pública y participación en la toma de decisiones vitales para grupos humanos multidiversos.

Hay ejemplos ya que demuestran la poca utilidad práctica de estas decisiones (Rodríguez y col. 2008). Nacen poblados dormitorios o de mudanza temporal, que en muchos casos terminan acogiendo a otras familias distintas a las reubicadas inicialmente por ser damnificadas, las que decidieron un retorno paulatino a sus poblados iniciales, en los que se reencuentran con sus condiciones históricas de modo de vida y donde recuperan sus subjetividades, roles, funciones y relaciones vinculantes.

La idiosincrasia del ser costero lo afianza a su terruño y a su forma diaria de sustento, que va conectado no solo al sustento económico sino también a los sentimientos, las relaciones interpersonales, el establecimiento y fortalecimiento de redes sociales muy interconectadas, robustas en el tiempo, 
en las que se afianza la solidez social e integridad de la comunidad y que son un elemento esencial a favor de su capacidad de resiliencia ante impactos ambientales y estresores externos.

Un enfoque estratégico a ser considerado em el trabajo con las comunidades es la percepción social de la valoración económica o vital de los recursos naturales, que construye el ser humano en su decursar histórico y que se transmite generacionalmente.

Los ecosistemas o sus fragmentos, si se aborda la temática por estudios de caso, no tienen una igual valoración entre espacios similares o entre espectadores/actotres semejantes, pues su valor no radica única ni priciplamente en la sumatoria de sus componentes y usos, si no en el conglomerado de experiencias, sentimientos, afectos y desafectos que el ser humano va construyendo e comunidad, temporal y fisicamente, en su vínculo con los recursos naturales.

Hay una variable de fuerte componente sócio-cultural que no está siendo muchas veces considerada, pues los análisis de impacto, desarrollo, vulnerabilidad, adaptación y mitigación tienen un basamento mayoritariamente biogeográfico y se desestima $o$ no se profundiza suficientemente en las interioridades sociales del intercambio del ser humano con el espacio común, natural o construido.

En este sentido hay una alerta importante a considerar o se seguiría subvalorando el aspecto subjetivo del hombre insular y su vínculo originario con su entorno, proceso en el que quedan huellas de ambas partes.

El riesgo de intercatuar con las comunidades costeras con un enfoque sesgado en este sentido, nos conduce al diseño de programas y acciones de adaptación al cambio climático que se basan con mayor balance en temáticas de rehabilitación de ecosistemas, recuperación de infraestructuras o relocalización de poblados, sin considerar suficientemente los riesgos sociales como un elemento piramidal en la búsqueda de las soluciones mas atinadas y coherentes. Estas situaciones se dan a lo largo de la islã, aún cuando los escenarios son diferentes desde el punto de vista socioeconómico y geográfico. 
Cuba, tiene una línea costera irregular. En varias zonas es una gran plataforma baja que se extiende desde el litoral de bosques de manglar hasta el borde submarino de la plataforma insular o veril, interceptada en varios sitios por cinturones de cayos formados mayormente por manglar, rocas y arrecifes de coral.

Las zonas más reconocidas de esta estructura, que generan grandes lagunas interiores, denominada en la toponimia como bahías, son los Archipiélagos conocidos como Jardines del Rey al norte y Jardines de la Reina al Sur, en la región central del país.

Otras zonas litorales son, por el contrario, de costa rocosa acantilada, como la de Santiago de Cuba, Baracoa, la región entre Playa Ancón en Santi Spiritus hasta Bahía de Cochinos en Matanzas o el norte de La Habana, por poner ejemplos.

Hay una diferencia notable entre las vulnerabilidades de estos particulares escenarios. Las zonas bajas de bahías interiores son altamente proclives al impacto de la surgencia del mar y a la elevación de su nivel medio, que penetra en forma de cuña salina por sus cuencas y suelos fértiles, cubriendo terrenos costeros que van perdiendo sus capacidades para la agricultura y los cultivos en general.

A esta situación se suma la construcción de viales sobre el mar para acceder a cayos de espectaculares valores paisajísticos para el desarrollo del turismo, pero que han modificado las corrientes y el ritmo de las mareas interiores.

En cambio, las costas rocosas representan un rompeolas natural y un retenedor y disipador de energía del mar. Se convierten en un obstáculo para la penetración del mar. En estas costas se hace menos notable la penetración marina y de la cuña salina tierra adentro, aunque sus ríos también son penetrados por el océano, tanto em sus cause como e las orillas, que van perdiendo condiciones para el cultivo agrícola.

Vinculado a estos elementos, esencialmente geográficos, está la vulnerabilidad de las pequeñas comunidades costeras del país, normalmente con pocos habitantes permanentes (incrementados en temporada de verano) y 
que no alcanzan la complejidad estructural y de servicios comunitarios que las clasifique como ciudades.

La tipología constructiva de estas pequeñas comunidades costeras es por lo general de una arquitectura ligera, que busca el fresco interior, la entrada de la luz y a la vez la protección del sol con soleras y amplios portales. Son poblados de calles anchas y en algunos casos mantienen construcciones sobre pilotes en el mar. La madera es el material constructivo más recurrente, así como techos ligeros de diferentes materiales.

Sin embargo, en los poblados que se vinculan directamente o por la vía de mano de obra a sectores turísticos establecidos o en crecimiento, mejoran en poco tiempo su tipología constructiva, buscando soluciones más duraderas a la intemperie y el accionar constante del spray salino. Indicador evidente de solvencia y mejora económica que permite mayor bienestar familiar, mejoramiento de la calidad de vida y disminución de la vulnerabilidad estructural.

La Agencia de Medio Ambiente del Ministerio de Ciencia Tecnología y Medio Ambiente de Cuba, en conjunto con otras instituciones de ciencia del país ha venido implementando el Macroproyecto. "Escenarios de peligro y vulnerabilidad de la zona y vulnerabilidad de la zona costera cubana asociados al ascenso del nivel medio del mar para 2050 y 2100" que tuvo un cierre de resultados de una primera etapa en 2015 en formato de Mapa Informe Nacional.

Este estudio a escala país se basó en evaluaciones in situ de diferentes variables, cuyo análisis y comparación permitieron pronosticar impactos futuros en dos escenarios temporales: al 2050 y 2100.

Se estudiaron 122 asentamientos costeros alrededor de la isla, aportando resultados preocupantes desde todo punto de vista. Para el primer escenario temporal se modela en base a 0,27 $\mathrm{m}$ de elevación del nivel medio del mar y para el segundo en base a 0,85 m.

Se prevé entonces que para el 2050 trece asentamientos tengan afectación total por penetración permanente de las aguas, impactando directamente a 6655 habitantes y 98 poblados se verán con afectación parcial 
y anegaciones temporales. Las afectaciones totales a la vivienda llegan a las 4 176 casas inundadas.

Para 2100 se sumarían seis comunidades costeras con afectación total y 1956 personas, además de 99 afectadas parcialmente. Se inundan con afectación total 966 viviendas (Imagen 1).

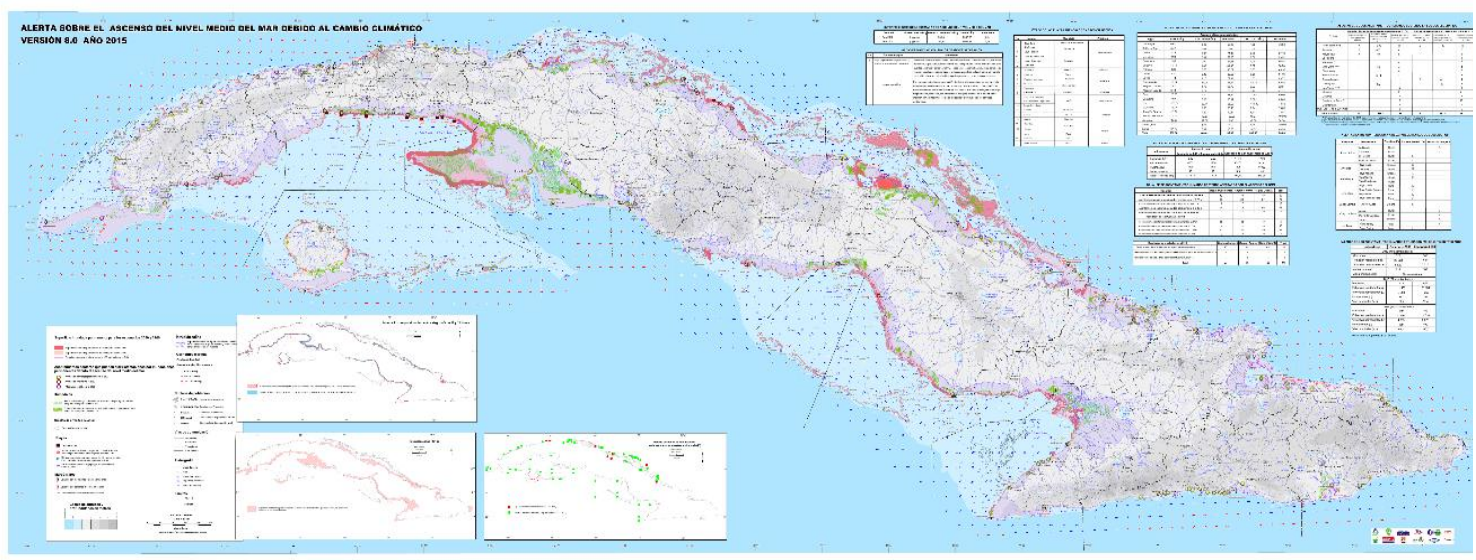

Imagen 1. Alerta sobre elevación del nivel medio del mar. Escenarios 2050-2100. Fuente: Macroproyecto de Zonas Costeras, AMA 2015.

Entre los aspectos más complejos a definir está entonces la relocalización previa de estas pequeñas comunidades costeras que serán o ya están siendo penetradas por el mar hacia zonas más elevadas y alejadas de la costa.

Ante este fenómeno de desplazamiento causado por el clima cambiante se establecen decisiones comunitarias de permanecer en sus lugares originarios, basadas en fuertes condicionamientos históricos, vitales y de idiosincrasia marino-costera.

Como paliativo o alternativa a este fuerte impacto social de la relocalización planificada pueden aparecer opciones ingenieras o de soluciones blandas fundamentadas en la recuperación y el fortalecimiento de los ecosistemas naturales en adaptación a los nuevos momentos y funciones ambientales de los ecosistemas rehabilitados.

De igual forma, se valora la pertinencia de una arquitectura para la adaptación y mitigación de los efectos del cambio del clima en pequeñas comunidades costeras. 


\section{EL HÁBITAT DE LAS PEQUEÑAS COMUNIDADES COSTERAS Y SU SOSTENIBILIDAD}

El espacio antropizado en su doble dimensión: física y simbólica constituye una mediación esencial en la conformación de la comunidad al caracterizar determinadas formas de relaciones sociales en las que la naturaleza de los vínculos establecidos entre los sujetos sociales y con el sitio son expresión de una determinada cosmovisión: de una cultura.

La acelerada urbanización como consecuencia del crecimiento de la industria, del sector terciario y de la reconfiguración "digital" de las formas de socialización ha incrementado las brechas de accesibilidad universal entre los polos urbanos de mayor y de menor atractividad. Ello ha acentuado las migraciones escalonadas, los desplazamientos de población y ha elevado desproporcionadamente las demandas urbanas, así como los impactos negativos de la ciudad sobre el medio ambiente.

El desarrollo humano confronta su efecto global en términos de cambio climático. Las políticas públicas afines se debaten entre: su afrontamiento, o la adaptación(Pacheco-Mangas y Palma-García, 2015). En este contexto ganan terreno las teorías sobre la resiliencia social entendida como "el estudio de las respuestas y la adaptación positiva de personas que viven en entornos de riesgo, en contextos y situaciones que suponen amenazas potenciales al desarrollo" (Uriarte Arciniega, 2014)

En Cuba, los estudios sobre resiliencia han sido vinculados a los de prevención de "peligro, vulnerabilidad y riesgos" (PVR) sustentados en la premisa de "reducir y evitar las pérdidas humanas" (Muñiz, 2018).

Su expresión está orientada desde el Instituto de Planificación Física y se focaliza en los asentamientos humanos costeros. Como resultado fue aprobada, en 2017, la "Tarea Vida", un Plan de Estado para el Enfrentamiento al Cambio Climático, que reúne las acciones de adaptación y mitigación 
dirigidas a la creación y acondicionamiento de asentamientos humanos cada vez más resilientes ${ }^{4}$. (Muñiz, 2018)

Diversos son los aspectos destacables de la perspectiva cubana de resiliencia urbana: su proyección de encargo político del Estado, su visión estratégica capaz de jerarquizar entre los fenómenos que más afectan la estabilidad del sistema de asentamientos humanos y de la economía, su sentido humanista enfocado a salvaguardar la vida humana como prioridad, y su enfoque de proceso permanente.

Sin embargo, los elevados niveles de centralización, de verticalismo y de asistencialismo, unido a la insuficiencia e ineficiencia de los canales de participación coartan el involucramiento de los sujetos sociales a nivel comunitario en la mitigación, adaptación y afrontamiento de las problemáticas descritas. Aún más, toda vez que el tratamiento del asunto implica, como solución, el desplazamiento de los asentamientos humanos costeros.

Las acciones de repliegue frente a las modificaciones medioambientales no constituyen un ejemplo de adaptación. El distanciamiento del problema conduce a considerarlo resuelto enajenando al sujeto social de la posibilidad real de contribuir a su superación. A la vez, al quebrar el vínculo comunitario se imposibilita el aprendizaje colectivo de los sujetos sociales para su afrontamiento y mitigación. Se desconoce por tanto que “...las personas y las comunidades poseen mecanismos de recuperación... [entre las que se] ...incluyen la eficacia colectiva, la cultura ciudadana y/o la resiliencia comunitaria. (Ruiz Pérez, 2015)

Los estudios de resiliencia en Cuba están planteados desde principios de resiliencia urbana ${ }^{5}$. Sin embargo, las prácticas de reubicación de asentamientos costeros, aunque pensadas desde la seguridad ciudadana obvian la dimensión cultural del territorio y las nefastas consecuencias de la

\footnotetext{
${ }^{4}$ Se han concluido estudios técnicos en cuatro municipios cubanos (Santiago de Cuba, Bayamo, Guanabacoa y Centro Habana) con el objetivo de elevar la resiliencia urbana de las principales ciudades en Cuba.

${ }^{5}$ Que enfatizan en la capacidad de la ciudad para resistir una amenaza, para absorber, adaptarse y recuperarse de sus efectos de manera oportuna y eficiente, lo cual incluye la preservación y restauración de sus estructuras y funciones básicas.
} 
ruptura de la relación entre el hombre y su medio como asiento, como espacio de subsistencia, como

cultura y como patrimonio. El traslado y reubicación de la población trae consigo múltiples impactos negativos:

- El quiebre de las relaciones sociales y los vínculos comunitarios; la ruptura de la relación hombre - medio;

- La deslocalización de asentamientos humanos ajenos a la actividad económica de sustento y a los elementos originarios de su ubicación;

- La alteración de la dinámica social, y el desarraigo cultural.

Al profundizar en la relación hombre - medio, ya sea desde la práctica social cotidiana o valorativa, se ahonda en la interpretación del "lazo afectivo establecido entre las personas y el lugar" (Tuan, 1974; Árias y Ramírez, 2013; Constain, 2012). Es esta una perspectiva que ayuda a la comprensión dialéctica del "territorio - patrimonio cultural"; de acuerdo con la cual constituye un destacable campo simbólico, manejado y modificado constantemente por la sociedad, por tanto, manifestación de la identidad colectiva de quienes lo habitan. (Delgado, 2010: 27)

El abordaje de la resiliencia para un desarrollo sostenible desde una visión cultural del territorio supone la búsqueda de formas simbióticas de convivencia entre la comunidad y el medio. al respecto se aviene mejor la definición de resiliencia socio-ecológica aportada por De La Torre-Valdez y Sandoval-Godoy (2015) quienes la comprenden como "la capacidad de adaptación que desarrollan grupos sociales al lograr un equilibrio territorial a medida que su contexto ecológico se transforma". En esta perspectiva la aspiración al desarrollo sostenible desde un enfoque de resiliencia social, comunitaria y territorial es inconsistente sin una cultura y una educación del territorio.(Baroni, 2003:74)

Un enfoque de resiliencia social comunitaria implica desarrollar las potencialidades para el aprendizaje colectivo. Este posibilitará, en términos de autodesarrollo comunitario, una conciencia crítica entorno a la problemática, así como la movilización hacia la participación colectiva y cooperada en el proyecto de adaptación y afrontamiento a los efectos del cambio climático. Ello 
posibilitará, en armonía con la propia transformación del medio reajustar la organización social, las formas de ocupación del espacio, las técnicas y tecnologías constructivas, el modo de vida, y las prácticas socioculturales, incluidas las prácticas productivas.

En ella los sujetos sociales comunitarios son los artífices de su propio desarrollo social, desde las potencialidades endógenas, en relación con el territorio e insertos en el entramado socio-territorial, de modo que la cultura territorial, así como los saberes constituidos en colectivo y las relaciones sociales históricamente construidas sean dispuestos para la transformación social. La resiliencia social comunitaria implica el fortalecimiento del estado de comunidad como cualidad del desarrollo desde la gestión del conocimiento como capital cultural; e implica su consolidación como comunidad de aprendizaje ${ }^{6}$.

La sostenibilidad del desarrollo en Cuba desde una perspectiva de resiliencia social comunitaria ha de pasar por atender la vulnerabilidad urbano territorial expresada por medio de las contradicciones manifiestas en los conflictos: entre espacio urbano y rural, entre centro y periferia, entre los procesos de conservación y renovación, entre los procesos de desarrollo y de participación, de movilidad y accesibilidad universal, de envejecimiento poblacional, de seguridad social, de garantías de la fuerza laboral, y de sostenibilidad socio-cultural.

Como en gran parte del mundo, la vivienda continúa siendo un tema a resolver en la agenda de gobierno e institucional cubana. A los grandes déficits acumulados se une el deterioro del fondo edificado, así como el crecimiento espontáneo y de baja calidad.

En el marco de las transformaciones socioeconómicas actuales el Estado ha dejado de ser el mayor proveedor de viviendas y favorece la política de construcción por esfuerzo propio y la construcción de células básicas mediante subsidios individuales a un sector en desventaja social. El resultado

\footnotetext{
6 "modelo educativo basado en los principios y prácticas de inclusión, igualdad y diálogo" (Serradell y Racionero, 2005) "que tiene por objetivos la transformación social y educativa" (Díez y Flecha, 2010), desde la "educación formal -escolarizada- y no formal -no escolarizada- como estrategia para (...) generar las bases de una convivencia solidaria". (Rodrigues de Mello, 2011; Elboj y Oliver, 2003)
} 
es la proliferación de viviendas de baja calidad funcional y estética que comprometen la conservación de la ciudad y su imagen, a la vez que la calidad de vida.

La tradición latinoamericana de diseño participativo de Livingston, Aravena permite sustentar que la cooperación y el involucramiento de la comunidad posibilita una intervención social del espacio urbano que permite: la recuperación del fondo edificado a través de viviendas mejor adaptadas a las necesidades y cultura locales; la construcción colectiva del espacio público; el empleo de tecnologías y tipologías adecuadas, la implementación de mejores formas de ocupación del espacio atenidas a la cultura territorial yel desarrollo de formas de gestión del hábitat local más eficientes.

\section{CONCLUSIONES}

Asumir la participación como proceso a través de su vínculo con el proyecto que le da direccionalidad permite 1) la incorporación de la temporalidad como dimensión analítica 2) una diferenciación del encargo que corresponde a cada sujeto implicado; 3) establecer una tipología de las etapas y la condición de participación que muestra el estado de simetría social presente en el proceso; y 4) la conformación de dos distribuciones, una empírica de la participación realmente existente en el proceso estudiado y otra teórica desde la lógica de la actividad social de que se trate cuya salida práctica aporta capacidad valorativa respecto al diagnóstico realizado y propositiva para la solución de problemáticas con el involucramiento de todos los que deban estar implicados.

La resiliencia de las comunidades costeras de Cuba ante los efectos del cambio climático tiene un componente esencial en la gestión integrada de las vulnerabilidades ambientales, estructurales y sociales, donde los actores esenciales implicados son tanto los habitantes de estos escenarios como los tomadores de decisiones que gestan e implementan las accioones del desarrollo. 
La resiliencia social comunitaria implica desarrollar potencialidades para el aprendizaje colectivo, favoreciendo la creación de un habitat que tanto asegure la subsitencia de las poblaciones imbricadas en los ambientes naturales como que genere espacios de coexistencia de redes sociales fuertemente arraigadas a su cultura histórica. Una parte significativa del esfuerzo exitoso de gestión para el desarrollo en zonas de alto riesgo, se basa en la disminución de las vulnerabilidades del habitat via la inclusión en la toma de decisiones. 


\section{REFERENCIAS}

Alonso Freyre, Joaquín. \& Jara Solenzar, Dunia. (2016) "Participación diferenciada en proyectos de desarrollo" en Simposio Internacional CIPS 2016. La Habana: Convención Internacional sobre Ciencia, Tecnología e Innovación. CITMA.

Árias, S. \& Ramírez, C. (2013). Usos y abusos del paisaje cultural cafetero: una reflexión desde el concepto de patrimonio. Revista Jangwa Pana, 12 (enero diciembre), 115-128

Baroni Bassoni, S. (2003). Hacia una cultura del territorio. Centro Regionale de Intervento per la Cooperazione (CRIC) - Grupo para el Desarrollo Integral de la Capital. Ciudad de La Habana.

Bourdieu, P. (1988) Cosas dichas. Buenos Aires: Gedisa.

Caballero Rivacoba, M. T. \& Yordy García, M. J. (2004): El trabajo comunitario: alternativa cubana para el desarrollo social. Camagüey: Ediciones Ácana y Universidad de Camagüey.

Colectivo de autores (2016) De lo superficial a lo esencial. Lo comunitario en prácticas culturales. La Habana: Instituto Cubano de Investigación Cultural Juan Marinello.

Colectivo de Autores, 2015. Macroproyecto "Escenarios de peligro y vulnerabilidad de la zona costera cubana asociados al ascenso del nivel medio del mar para 2050 y 2100" Editorial Academia, 54 pp.

Constain, J. C. (2012). Análisis de la incorporación del Patrimonio Cultural Inmaterial en el Ordenamiento Territorial. Una propuesta de mecanismos de diálogo e inclusión para el caso de la Semana Santa de Popayán. Tesis en opción al Título de Profesional en Gestión y Desarrollo Urbanos, Universidad Colegio Mayor de Nuestra Señora del Rosario.

De La Torre-Valdez, H.C. \& Sandoval-Godoy, S. A. (2015). Resiliencia socioecológica de las comunidades ribereñas en la zona Kino-Tastiota del Golfo de California. Ciencia Pesquera, 23(1): 53-71

Del Río Hernández, M. (2002): La participación popular en el proceso de toma de decisiones públicas en el ámbito local comunitario en Cuba. Su régimen jurídico. Tesis doctoral. La Habana: Universidad de La Habana, Facultad de Derecho.

Delgado, A. E. (2010) El senderismo como instrumento de reconocimiento y revaloración del patrimonio de montaña, Chile, Fundación Sendero de Chile.

Díez, J.; Flecha, R. (2010). Comunidades de Aprendizaje: un proyecto de transformación social y educativa. Monográfico sobre Comunidades de 
Aprendizaje. Revista Interuniversitaria de Formación del Profesorado continuación de la antigua Revista de Escuelas Normales. 67, 24. http://www.aufop.com/aufop/uploaded_files/revistas/1268689288.pdf

Dilla, H.; González, G. \& Vicentelli, A. T. (1993): Participación y desarrollo en los municipios cubanos. La Habana: Editorial CEA.

Durán Castellón, Ginley. (2018) Participación de la comunidad portadora en la gestión patrimonial del centro histórico urbano de Remedios. Tesis doctoral. Santa Clara. UCLV, Centro de Estudios Comunitarios.

Elboj, C. y Oliver, P. (2003). Las comunidades de aprendizaje: Un modelo de educación dialógica en la sociedad del conocimiento. Revista Interuniversitaria de Formación del Profesorado. 17, 3, 91-103. http://aufop.com/aufop/uploaded_files/articulos/1219347435.pdf

IPCC, 2013: Climate Change 2013; The Physical Science Basis. Contribution of Working Groups to the Fifth Assesment Report of the Intergovermental Panel on Climate Change. [Stocker,T.F, D.Qin, G.K. Plattner, M, Tignor, Sk. Allen, J. Boschung, A. Nuels, Y. Xia, V.Bex, and P.M. Midgley (eds.)] Cambridge University Press, United Kingdom and New York, NY, USA, 135 pp.

Jara Solenzar, D. (2015) Expresión de la participación en la tradición parrandera en Chambas. Tesis doctoral. Santa Clara. UCLV, Centro de Estudios Comunitarios.

Juanes, J.L y col. 2014. Informe de Proyecto. MACROPROYECTO: Escenarios de peligro y vulnerabilidad de la zona costera cubana, asociados al ascenso del nivel medio del mar para los años 2050 y 2100 . PROYECTO 6. Actualización y evaluación de la evolución de las playas para los años 2050 y 2100. 35pp.

Muñiz González, A. (2018). Ciudades cubanas más resilientes, un desafío para el siglo XXI. Conferencia, Convención de Ordenamiento Territorial y Urbanismo 2018, Instituto de Planificación Física (IPF) palacio de Las Convenciones, La Habana

Pacheco-Mangas, J. \& Palma-García, M. de las O. (2015). La resiliencia en Servicios Sociales Comunitarios: un abordaje desde la perspectiva de los profesionales. I revista internacional de trabajo social y bienestar, № 4 (29-38)

Planos, E, Vega y R.A, Guevara. Editores, (2013). Impacto del Cambio Climático y Medidas de Adaptación en Cuba. INSMET, AMA, CITMA, Cuba 430 pp.

Rodrigues de Mello, R. (2011). Comunidades de aprendizaje: Democratización de los centros educativos. Recuperado a partir de https://repositorio.uam.es/xmlui/handle/10486/6363 
Rodríguez, E y col. 2008. Desarrollo comunitario en Isabela de Sagua. Informe de Proyecto Territorial 0640. CESAM, CITMA, 27pp.

Ruiz Pérez, J.I. (2015). Resiliencia comunitaria: propuesta de una escala y su relación con indicadores de violencia criminal. Pensamiento Psicológico, Vol. 13, No. 1, 2015, pp. 119-135. doi: 10.11144/Javerianacali.PPSI13-1.rcpe

Serradell, O., y Racionero, S. (2005). Antecedentes de las comunidades de $\begin{array}{llll}\text { aprendizaje. } \quad \text { Educar } \quad \text { (Bellaterra, } & \text { Spain), }\end{array}$ http://www.erevistas.csic.es/ficha_articulo.php?url=oai:educar.revistes.uab.cat: article/211\&oai_iden=oai_revista194

Tuan, Y.F. (1974). Topophilia: a study of environmental perception, attitudes, and values. Prentice-Hall, Englewood Cliffs, NJ.

Uriarte Arciniega, J.de D., (2014). Resiliencia y Envejecimiento. European Journal of Investigation in Health, Psychology and Education, 4 (2), 67-77.

Cibercultura y educación ambiental, un ejercicio en la formación de ciudadanos 